Boise State University

ScholarWorks

2-5-2009

Separation of Topographic Features from Magnetic Force Images Using Capacitive Coupling Effect

Byung I. Kim

Boise State University 


\title{
Separation of topographic features from magnetic force images using capacitive coupling effect
}

\author{
Byung I. Kim ${ }^{\mathrm{a})}$ \\ Department of Physics, Boise State University, Boise, Idaho 83725, USA
}

(Received 6 October 2008; accepted 11 January 2009; published online 5 February 2009)

\begin{abstract}
Separation of topographic features from magnetic images has been an issue for the past two decades in magnetic force microscopy (MFM). The frequent pickups of the topographic features are interpreted as transitions of the tip between bistable states of the tip-sample assembly in the noncontact and tapping regions. MFM using electrostatic force modulation demonstrates the separation of the topographic features from the magnetic images by removing the tapping state from the bistable states through the introduction of a capacitive coupling. () 2009 American Institute of Physics. [DOI: 10.1063/1.3077151]
\end{abstract}

\section{INTRODUCTION}

Separation of magnetic and topographic signals in magnetic force microscopy (MFM) has been a long-standing issue since its development 20 years ago. ${ }^{1}$ Although MFM is one of the most important imaging tools ${ }^{2}$ of nanoscale magnetic structures such as interspin interactions, ${ }^{3}$ vortex ratchets and cores, ${ }^{4,5}$ carrier-controlled ferromagnetism, ${ }^{6}$ and superconducting vortices, ${ }^{7}$ this issue still remains largely unsolved and thus has limited the current capability of the MFM as a quantitative magnetic imaging tool. ${ }^{2,8}$ The existing separation methods have focused on monitoring magnetic signals as the tip follows the sample topography. Schönenberger and co-workers ${ }^{9,10}$ developed a MFM technique that monitors the dc-magnetic force (magnetic image) under the constant amplitude (topography image) between the sample and the tip. The technique gives reasonably good separation of topographic and magnetic information. Later, Giles et al. ${ }^{11}$ made an important contribution to the separation of the two signals through the development of a twopass technique, the so-called "tapping/lift mode" MFM. In this technique, the first scan is for the topographic signal and then the second scan is repeated to record magnetic information (either as variations in amplitude, frequency, or phase of the cantilever oscillation) in the same line scan at a constant elevated height above the surface. The technique is good at eliminating topographic features in the magnetic image, but the tip contact to the sample during the tapping scan causes some problems. First, the tip-stray field can frequently and significantly disturb the magnetization distribution in a sample, especially in a soft magnetic material, during tapping. ${ }^{12}$

Second, the sharp magnetic tip may be easily worn in the presence of topographic variations on the sample surface. ${ }^{8}$ Third, the imaging mode places higher demands on instrument stability, or the continual alternating line scan causes serious reduction in the correlation between the two scans due to drift. $^{8}$ To minimize these problems, the topographic

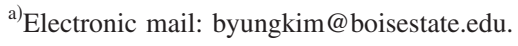

features need to be separated from MFM images without touching the sample surface, thus minimizing the influence of the tip-stray field on a sample magnetization. As we shall see below, however, magnetic images taken with the conventional noncontact amplitude modulation (AM) MFM frequently picked up topographic features even at larger distances $(100-200 \mathrm{~nm}){ }^{8,13}$ Here, the mixing mechanism of the two signals in the conventional AM MFM is identified using nonlinear dynamics between the tip and the surface. A method to separate them using a capacitive coupling of the electrostatic force modulation is proposed and verified.

\section{EXPERIMENTAL}

A heavily doped $\mathrm{Si}$ cantilever coated with a thin magnetic Co layer is used (Nanosensors) for the detection of the magnetic force. The force constant and the resonance of the cantilever are $2.1 \mathrm{~N} / \mathrm{m}$ and $106 \mathrm{kHz}$, respectively. The lock-in phase was set to make the driving signal be "in phase" with the response signal of the cantilever at the tip-sample distance greater than $1000 \mathrm{~nm}$ from the sample surface. The in-phase $\omega$ component amplitude $X_{\omega}$ $\left(=R_{\omega} \cos \phi_{\omega}\right)$ was measured as a function of tip-sample distance to understand its behavior. The in-phase amplitude is selected as a feedback signal because of its higher sensitivity with the tip-sample distance over the amplitude $R_{\omega}{ }^{14}$ The tip is magnetized by placing the cantilever in a magnetic field of $0.2 \mathrm{~T}$, aligned perpendicularly to the lever, for $3 \mathrm{~min} .{ }^{15} \mathrm{The}$ scan rate is $1 \mathrm{~Hz}$ and the time constant is set at $100 \mu \mathrm{s}$ in a lock-in amplifier (EG \& G Princeton Applied Research, MODEL 5302). A CoCr film deposited on the glass substrate by the dc magnetron sputtering method up to a thickness of $300 \mathrm{~nm}$ was used as a sample for this study. All the data shown here are obtained with a commercial AutoProbe LS (former Park Scientific Instruments) in air.

\section{TOPOGRAPHIC FEATURES IN A MAGNETIC FORCE IMAGE}

A magnetic tip is mechanically vibrated at the resonance frequency of the cantilever with the free oscillation ampli- 

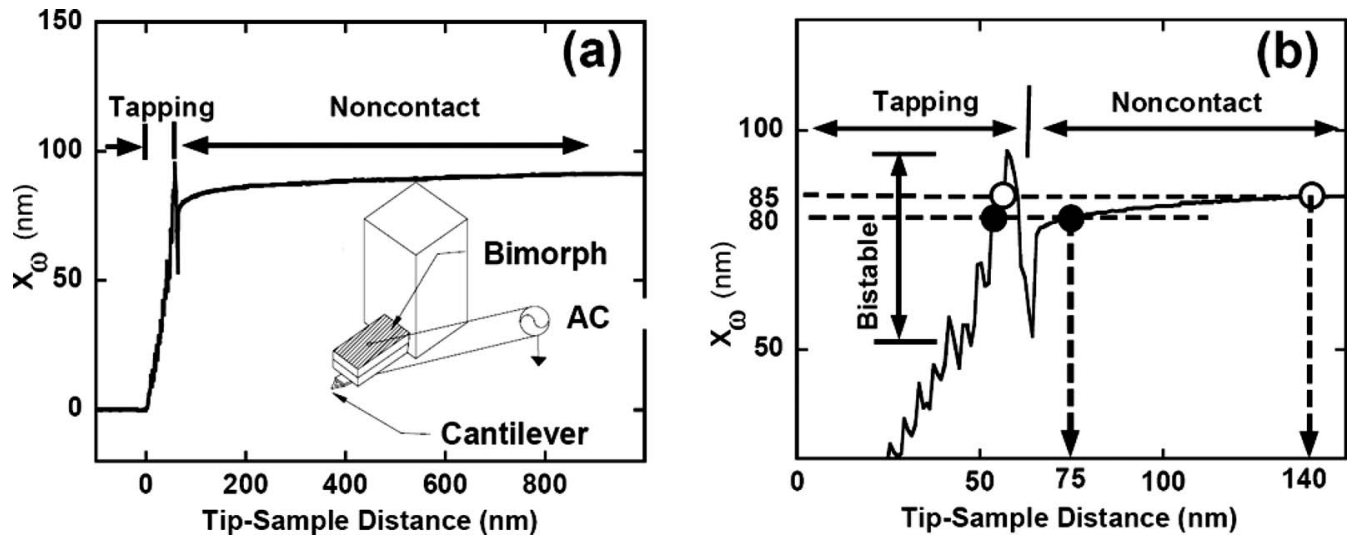

FIG. 1. (a) A typical in-phase amplitude vs distance curve at the operating frequency of resonance $106 \mathrm{kHz}$ and the free oscillation amplitude of $96 \mathrm{~nm}$ on a CoCr film. (Inset) a schematic of the acoustic excitation method. (b) An enlarged amplitude-distance curve in the distance range between 0 and $150 \mathrm{~nm}$. Horizontal dashed lines represent the feedback set amplitudes of 80 and $85 \mathrm{~nm}$ that correspond to the average noncontact distances of $75 \mathrm{and} 140 \mathrm{~nm}$ from the surface, respectively, as marked with dashed arrows. The two stable states are marked with two circles for each set amplitude.

tude of $96 \mathrm{~nm}$ by the acoustic excitation method such as the bimorph driven system [inset of Fig. 1(a)]. A dc bias voltage $V_{\mathrm{dc}}=+10 \mathrm{~V}$ is applied between the tip and the surface to create a long-range attractive electrostatic interaction, which is essential to make the feedback polarity stay constant, regardless of the attractive or repulsive magnetic forces for stable feedback. ${ }^{10,13}$ In the in-phase amplitude-distance curve, the amplitude decreases monotonically as the tip moves toward the surface for the distance between 64 and $1000 \mathrm{~nm}$ ("noncontact region") in Fig. 1(a). For magnetic imaging, the average tip-sample distance of the oscillating tip should be positioned in the noncontact region where the magnetic signal is dominant over the topographic signal. Since the sign of slope is positive (i.e., $\partial X_{\omega}(d) / \partial d>0$ ) in the noncontact region, it is necessary to set the feedback polarity to positive for stable magnetic imaging. The linear tapping region also has the same sign of slope, ${ }^{16,17}$ indicating that there exist two stable states for a given set amplitude $\left(\mathrm{X}_{\omega, \mathrm{SP}}\right)$, one in the tapping region and the other in the noncontact region.

The key approach of this paper is to solve a 20-year-old problem by using the fundamental understanding of nonlinear stochastic physics in the tip-sample interactions of AM atomic force microscopy (AFM) recently discovered and published by Garcia and San Paulo. ${ }^{18}$ Garcia and San Paulo associated abrupt changes in height of topographic features with the continual switching of the oscillating tip between the two stable states during the tip scanning over the surface in the AM AFM. Similarly, in this paper, the topographic features in the magnetic image are attributed to the switching between the bistable states. This effect is analogous to the sudden transition from the noncontact to contact states in contact mode AFM. ${ }^{19}$ In principle, both effects result from the intrinsic nonlinear mechanical bistability of the sensorsample assembly. In the contact AFM, the so-called "snapto-contact" issue has been resolved by removing the bistability using a voltage-activated force feedback. ${ }^{20,21}$ Similarly, in this paper, this issue has been addressed by removing the stable state in the tapping region. The bistability originates from the dc-bias voltage to induce monotonic decrease in the long-range amplitude as the distance becomes smaller in the noncontact region between 64 and $1000 \mathrm{~nm}$ [Fig. 1(a)].

In order to verify this concept, the magnetic imaging was performed repeatedly at two different set amplitudes $\left(X_{\omega, \mathrm{SP}}\right)$ of 80 and $85 \mathrm{~nm}$ at the same tip location. The average operating spacing can be determined graphically by projecting to the $x$-axis from the intersecting points where the open-loop curve and a feedback set point line meet, as shown in Fig. 1(b). The origin is chosen as the point where the linearextrapolated line in the tapping region crosses with the $x$-axis, and thus the $x$-coordinate represents the average tipsample distance. ${ }^{17}$ The average noncontact distances are determined to be 75 and $140 \mathrm{~nm}$ for the set amplitudes of 80 and $85 \mathrm{~nm}$, respectively. Figs. 2(a) and 2(b) show stripelike structures that correspond to the magnetic domain features because of a higher contrast variation at a larger tip-sample distance [see line scans in Figs. 2(a) and 2(b)], a well-known signature for magnetic features. ${ }^{22}$ Topographic hillocks and grains appear as spots with diameters of 1000-2000 nm and spots with diameters of 100-300 nm, respectively, in Fig. 2(a) [see more details in Fig. 4(b)]. Most of the topographic
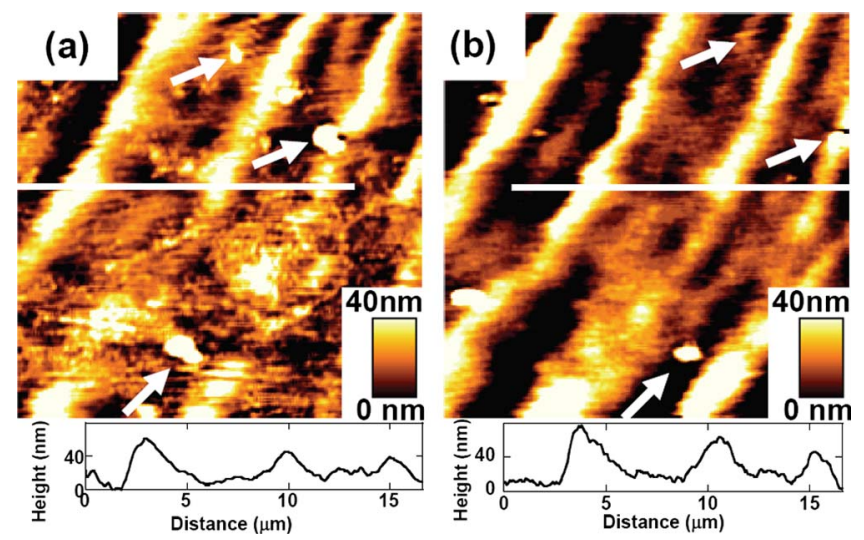

FIG. 2. (Color online) (a) A stripelike magnetic domain image with hillocks and magnetic grains on the $\mathrm{CoCr}$ film (scan area: $20 \times 20 \mu \mathrm{m}^{2}$ ). (b) The same stripelike magnetic domain image with sporadic hillocks with $4 \mu \mathrm{m}$ shift to the left from the position of (a) (scan area: $20 \times 20 \mu \mathrm{m}^{2}$ ). (Insets) Line scans along the white lines in each image for comparison of the contrast variation between two images. Common topographic hillocks in both images are as marked with arrows for comparison. 

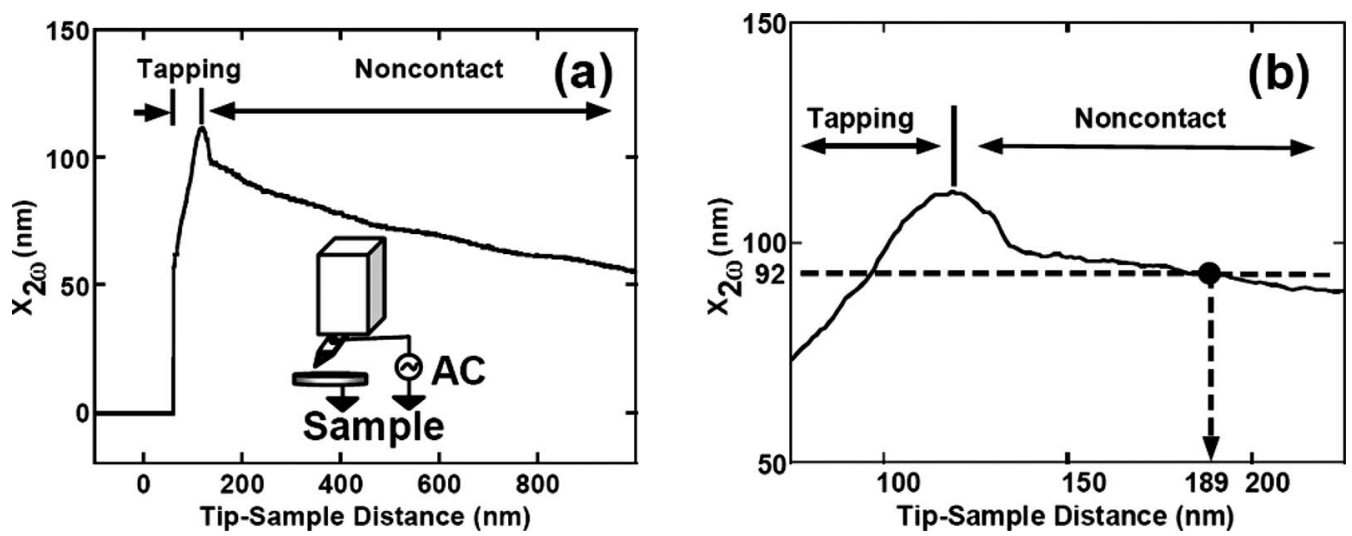

FIG. 3. (a) A typical in-phase amplitude vs distance curve at the operating frequency of resonance $53 \mathrm{kHz}$. (Inset) A schematic of the electrostatic force modulation method. (b) An enlarged amplitude-distance curve in the distance range between 75 and $225 \mathrm{~nm}$. A dashed line representing the feedback set amplitude of $92 \mathrm{~nm}$ corresponds to the average noncontact distance of $189 \mathrm{~nm}$ from the surface. A stable state is marked with a solid circle in the noncontact region.

features drastically disappear except a few hillocks marked with arrows in Fig. 2(b). The remarkable change in pickup ratio results from the noncontact lift-height change by $65 \mathrm{~nm}$ $(=140-75 \mathrm{~nm})$ in Fig. 1(b). In the constant amplitude mode, the average tip-sample distance continues to vary in order to maintain the set-amplitude constant. Because the setamplitude $80 \mathrm{~nm}$ is comparable to the average tip-sample distance of $75 \mathrm{~nm}$, feedback perturbations allow for switching between the bistable states for picking up topographic features. The switching between two stable states would be almost equally probable during the data acquisition explaining the pickup ratio of nearly one, as shown in Fig. 2(a). At the set amplitude of $85 \mathrm{~nm}$, the average noncontact distance of $140 \mathrm{~nm}$ is somewhat bigger than, but still smaller than, the heights of bigger hillocks of 200-300 $\mathrm{nm}$. The oscillating tip spends most of its time collecting magnetic features in the noncontact region except the regions of the bigger hillocks, explaining the small pickup ratio in Fig. 2(b). The bistable states exist for almost all of the average tip-sample spacing from 64 up to $>1000 \mathrm{~nm}$ in Fig. 1(a). This indicates that topographic features always have a chance to appear in a magnetic image, except for smooth and homogeneous sample surfaces.

\section{SEPARATION OF TOPOGRAPHY AND MAGNETIC STRUCTURES}

As a method to make the amplitude increase in the noncontact region as the tip approaches the surface, an electrostatic force modulation method is introduced to use the capacitive coupling effect for magnetic imaging [the inset of Fig. 3(a)]. The same rough surface is used for a direct comparison between both methods in separating topographic features from a MFM image. A sinusoidal signal $V_{\text {ac }} \sin (\omega t)$ with $V_{\mathrm{ac}}=10 \mathrm{~V}$ is applied between the same tip and the same surface using a function generator (Hewlett Packard, HP 33120A). The operating frequency $\left(f_{\text {op }}\right)$ was set to $53 \mathrm{kHz}$ where in-phase $2 \omega$ component amplitude $X_{2 \omega}$ $\left(=\mathrm{R}_{2 \omega} \cos \phi_{2 \omega}\right)$ has the resonance peak for the cantilever with the resonance frequency of $106 \mathrm{kHz} .{ }^{23}$ In Fig. 3(a), the in-phase amplitude increases in the noncontact region as the tip approaches the surface. The enlarged curve [Fig. 3(b)] shows that the horizontal set-amplitude line $\left(X_{2 \omega}=X_{2 \omega, \mathrm{SP}}\right)$ meets twice with the in-phase amplitude-distance curve, but the signs of the slope $\partial X_{2 \omega} / \partial z$ (i.e., the feedback polarity) at the two intersecting points are different from each other. The result shows that only one stable state (in either tapping or noncontact region) is available for a given feedback polarity.

Magnetic imaging of the same $\mathrm{CoCr}$ magnetic film was performed under the feedback condition of a constant set amplitude of $92 \mathrm{~nm}$ in the noncontact region to investigate the effect of the capacitive coupling on the separation. The imaging condition is similar to the conditions of Fig. 2(b) because the noncontact distance of $189 \mathrm{~nm}$ is much bigger than the set amplitude of $92 \mathrm{~nm}$, but still smaller than the heights of the hillocks. Fig. 4(a) shows a magnetic image of well-connected labyrinthine structures with the periodicity of 4.5-5 $\mu \mathrm{m}$. The image does not show any evidence of the topographic features. More magnetic images are collected repeatedly in different scan areas from $100 \times 100 \mathrm{~nm}^{2}$ to $40 \times 40 \mu \mathrm{m}^{2}$ for the different set values between 82 and 96 $\mathrm{nm}$ (not shown). However, neither image shows the topographic features, indicating that there is no mechanical contact between the tip and the surface during MFM data acquisition. The reproducibility of MFM data without tip crashing toward the surface suggests the enhanced stability of MFM method using the capacitive coupling over the conventional AM MFM. In order to observe the topographic features, the oscillating tip is brought into the tapping region by reversing feedback polarity with the same set amplitude of $92 \mathrm{~nm}$ [see
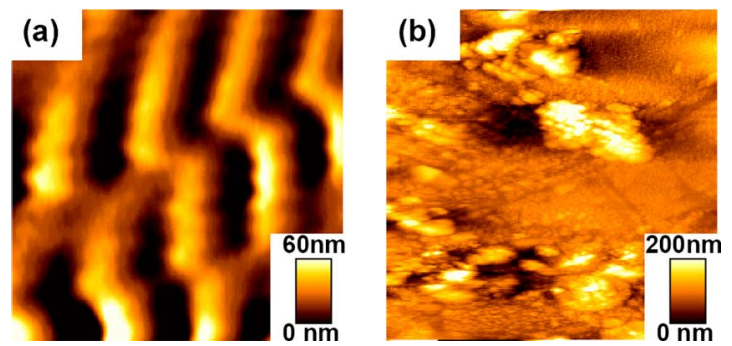

FIG. 4. (Color online) (a) The stripelike magnetic domain image in the noncontact regime with the set amplitude of $92 \mathrm{~nm}$ (scan area: 20 $\times 20 \mu \mathrm{m}^{2}$ ). (b) Topographic image of CoCr film taken with the electrostatic tapping mode with the set amplitude of $92 \mathrm{~nm}$ (scan area: $\left.20 \times 20 \mu \mathrm{m}^{2}\right)$. 


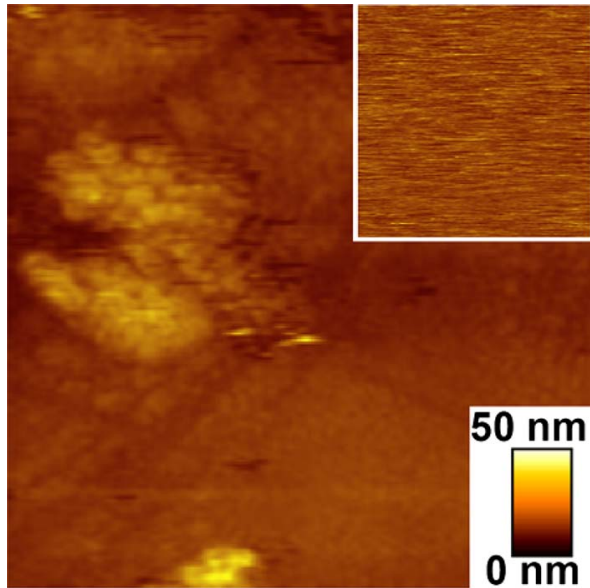

FIG. 5. (Color online) A topographic image taken by a nonmagnetic tip in the tapping regime with the set amplitude of $92 \mathrm{~nm}$ (scan area: 20 $\times 20 \mu \mathrm{m}^{2}$ ). (Inset) An image taken by the same nonmagnetic tip in the noncontact regime with the set amplitude of $92 \mathrm{~nm}$ (scan area: 20 $\left.\times 20 \mu \mathrm{m}^{2}\right)$

Fig. 3(b)]. Fig. 4(b) shows several hillocks with the diameters of 1000-3000 nm and heights of 200-300 nm and aggregations of small grains with the sizes of $100-300 \mathrm{~nm}$ and the heights of 10-40 nm, consistent with those observed in Figs. 2(a) and 2(b). The consistency indicates that the charging effect due to the electrostatic modulation is not usually important for general conductive magnetic samples. ${ }^{8}$

An image taken in a tapping regime by a nonmagnetic commercial conductive $\mathrm{Si}$ cantilever ${ }^{23}$ is shown in Fig. 5 where the topographic features are similar to those in Fig. 4(b). When an image was taken in a noncontact regime (inset of Fig. 5), it was completely featureless without showing any evidence of magnetic features within the current noise level of the system. The result indicates that topographic interactions (e.g., electrostatic force) are too weak to provide observable topographic features. It also suggests that the magnetic interactions should be dominant over the topographic interactions at a noncontact distance around $\sim 200 \mathrm{~nm}$ where the magnetic image of Fig. 4(a) was taken. Both nonmagnetic images again support the separation of the topographic features from the magnetic images through the removal of one stable state using the capacitive coupling. The novel approach presented in this paper should have a dramatic impact on not only the MFM field, but also other scanning probe microscopy fields such as electrostatic force microscopy and Kelvin probe microscopy. Furthermore, the technique is expected to allow for less invasive observation of superconducting vortex structures and soft magnetic structures by avoiding the sudden transition from the noncontact state to the tapping state in the conventional bimorph driven system, thus leading to a better understanding of the relationship between magnetic structures and topographic pinning sites such as grain boundaries.

The author thanks Mr. In Seon Lee (KAIST) for providing the $\mathrm{CoCr}$ magnetic thin film samples.

${ }^{1}$ Y. Martin and H. K. Wickramasinghe, Appl. Phys. Lett. 50, 1455 (1987).

${ }^{2}$ M. R. Freeman and B. C. Choi, Science 294, 1484 (2001).

${ }^{3}$ U. Kaiser, A. Schwarz, and R. Wiesendanger, Nature (London) 446, 522 (2007).

${ }^{4}$ C. C. de Souza Silva, A. V. Silhanek, J. Van de Vondel, W. Gillijns, V. Metlushko, B. Ilic, and V. V. Moshchalkov, Phys. Rev. Lett. 98, 117005 (2007).

${ }^{5}$ T. Shinjo, T. Okuno, R. Hassdorf, K. Shigeto, and T. Ono, Science 289, 930 (2000).

${ }^{6}$ J. Philip, A. Punnoose, B. I. Kim, K. M. Reddy, S. Layne, J. O. Holmes, B. Satpati, P. R. Leclair, T. S. Santos, and J. S. Moodera, Nature Mater. 5, 298 (2006).

${ }^{7}$ E. W. J. Straver, J. E. Hoffman, O. M. Auslaender, D. Rugar, and K. A. Moler, Appl. Phys. Lett. 93, 172514 (2008).

${ }^{8}$ S. Porthun, L. Abelmann, and C. Lodder, J. Magn. Magn. Mater. 182, 238 (1998).

${ }^{9}$ C. Schönenberger and S. F. Alvarado, Z. Phys. B 80, 373 (1990).

${ }^{10}$ C. Schönenberger, S. F. Alvarado, S. E. Lambert, and I. L. Sanders, J. Appl. Phys. 67, 7278 (1990).

${ }^{11}$ R. Giles, J. P. Cleveland, S. Manne, P. K. Hansma, B. Drake, P. Malvald, C. Boles, J. Gurley, and V. Elings, Appl. Phys. Lett. 63, 617 (1993).

${ }^{12}$ X. Zhu, P. Grütter, V. Metlushko, and B. Ilic, Phys. Rev. B 66, 024423 (2002); X. Zhu and P. Grutter, IEEE Trans. Magn. 39, 3420 (2003).

${ }^{13}$ P. Grütter, H. J. Mamin, and D. Rugar, Scanning Tunneling Microscopy II (Springer, New York, 1992), pp. 151-207.

${ }^{14}$ M. A. Pasler and P. J. Moyer, Near Field Optics (Wiley, New York, 1996), p. 125.

${ }^{15}$ B. I. Kim, J. W. Hong, J. I. Kye, and Z. G. Khim, J. Korean Phys. Soc. 31, s79 (1997)

${ }^{16}$ Q. Zhong, D. Inniss, K. Kjoller, and V. B. Ellings, Surf. Sci. Lett. 290, L688 (1993).

${ }^{17}$ F. Perez-Murano, G. Abadal, N. Barniol, X. Aymerich, J. Servat, P. Gorostiza, and F. Santz, J. Appl. Phys. 78, 6797 (1995).

${ }^{18}$ R. Garcia and A. San Paulo, Phys. Rev. B 61, R13381 (2000).

${ }^{19}$ E. Meyer, H. Heinzelmann, P. Grütter, Th. Jung, Th. Weisskopf, H.-R. Hidber, R. Lapka, H. Rudin, and H.-J. Güntherodt, J. Microsc. 269, 152 (1988).

${ }^{20}$ S. A. Joyce and J. E. Houston, Rev. Sci. Instrum. 62, 710 (1991).

${ }^{21}$ J. R. Bonander and B. I. Kim, Appl. Phys. Lett. 92, 103124 (2008).

${ }^{22}$ Y. Martin, D. Rugar, and H. K. Wickramasinghe, Appl. Phys. Lett. 52, 244 (1988).

${ }^{23}$ B. I. Kim, U. H. Pi, Z. G. Khim, and S. Yoon, Appl. Phys. A 66, s95 (1998). 